\title{
Modeling Dynamic Beach Objects Using Spatio-Temporal Ontologies
}

\author{
D. van de Vlag ${ }^{1,2^{*}}$ and A. Stein ${ }^{1}$ \\ ${ }^{1}$ ITC, Department of Earth Observation Science, Hengelosestraat 99, 7500 AA Enschede, The Netherlands \\ ${ }^{2}$ ARCADIS Ruimtelijke Informatie, Het Rietveld 59a, 7321 CT Apeldoorn, The Netherlands
}

\begin{abstract}
This study models geospatial landscape features using spatio-temporal ontologies. A spatio-temporal ontology explicitly specifies a dynamic conceptualization of real world objects. Spatio-temporal ontologies are applied to the Isle of Ameland, relating beach nourishment to elevation, vegetation and wetness. The spatio-temporal ontology contains full membership functions for crisp objects, partial membership functions for fuzzy objects and temporal membership functions for dynamic fuzzy objects. The temporal membership functions include seasonal changes of vegetation and daily changes in wetness. Quality elements are discussed by means of a quality matrix, where ontological features (i.e. objects, attributes, relationships) are projected against their qualities. A sensitivity analysis showed that the calculated beach nourishment volumes are practically insensitive in relation to assumptions on the temporal membership functions. Spatio-temporal ontologies are shown to extend spatial ontologies to model dynamic processes in environmental studies in a more realistic way.
\end{abstract}

Keywords: Ameland, beach objects, ontologies, quality, sand suppletion, space-time

\section{Introduction}

Landscape objects are often dynamic. Typical examples include the beaches and dunes under coastal morphodynamics (Eleveld, 1999), agricultural fields in a rural-urban environment changing under population pressure (Marceau et al., 2001) and soil and geomorphological objects under shifting cultivation in Cameroon (Yemefack, 2005). The spatio-temporal datasets may be helpful for monitoring these dynamic landscape objects. Multi-source geo-information obtained by using satellite imagery is increasingly used to provide these sets. Satellite images supply basic measurements of biological and physical characteristics of the landscape objects, such as their positions, shapes, elevations, colors, temperature and moisture contents (Wilkie and Finn, 1996). A combination of images obtained at several instances may be useful for monitoring purposes. In this respect, remote sensing can assist policy makers, resource users and resource managers.

A typical aspect of landscape features is that they are frequently vague, both in their definition and in their spatial extent (Fisher et al., 2005). The main reasons are that both context and definitions are poor. Also, the objects are often delineated by conceptual ideas rather than by actual and quantifiable spatial extent.

Combination of different data sources can be difficult, because of different resolutions, spectral decomposition and sensor characteristics. To achieve information exchange between different data sources, the study of ontologies may unify different conceptualizations of geographical space into one

\footnotetext{
* Corresponding author: d.e.vlag@arcadis.nl
}

geographical ontology (Kokla and Kavouras, 2001). An ontology can be defined as an explicit specification of a conceptualization (Gruber, 1993). To do so, classes of objects are defined, including their relations and functions. Ontologies are hence characteristics for specific domains.

In the past, ontologies have proven to be useful for handling real world features within a geographical information system (Jeansoulin and Wilson, 2002; Van de Vlag et al., 2005). For each specific domain, ontologies identify and define a set of relevant concepts that characterize a given application domain. In this sense, domain ontologies reduce concepttual and terminological confusion. They also support interoperability and knowledge sharing within various government organizations (Jeansoulin and Wilson, 2002). Here, the decision makers demand certain data (Foody and Atkinson, 2002), whereas the uncertainty is inherent in spatial information. In recent scientific research on spatial information, uncertainty and spatial data quality in general are emphasized (Shi et al., 2003; Frank and Grum, 2004; Van Oort, 2006). Ontologies help to understand the role of the quality of the data sources as well as their fitness for use by decision makers (Hunter, 2001). So far, ontologies have been primarily applied in the spatial domain. For analyzing and understanding dynamic geographical problems, however, a spatio-temporal ontology, i.e. an ontology representing space and time, is essential (Frank, 2003a,b).

The objective of this paper is to define and apply a spatio-temporal ontology for modeling the dynamic landscape features. Such an ontology allows us to integrate the different multitemporal data sources. Its definition is based on an extension of the spatial ontologies with temporal issues, such as processes and events. We apply this spatial-temporal ontology 
to a beach management problem in the Netherlands, where because of erosion, beach nourishments have to be carried out. We will compare three methods. First, beach areas suitable for nourishment are represented and modeled by crisp objects. Second, they are represented and modeled by the fuzzy sets. Third, inclusion of a time series allows us to represent beach processes and events. A sensitivity analysis in the end validates choices in spatial and temporal fuzzification.

\section{Methodology}

To understand the characteristics of landscape features in time, an appropriate conceptualization of a spatio-temporal dataset is needed. To do so, we modeled landscape features by monitoring activities (Figure 1). Five operations are distinguished:

1. An ontological approach, which serves as a guideline for an explicit conceptualization of the landscape features.

2. Implementation of fuzzy rules, to describe the spatial and thematic vagueness.

3. Time correction, to correct for temporal variation of the attributes.

4. A quality matrix, to relate ontological features with several quality elements (e.g. positional-, thematic-, and temporal accuracy, completeness, etc.).

5. Model testing, by means of a sensitivity analysis.

In the following sections, a more detailed description on each of these five operations is given.

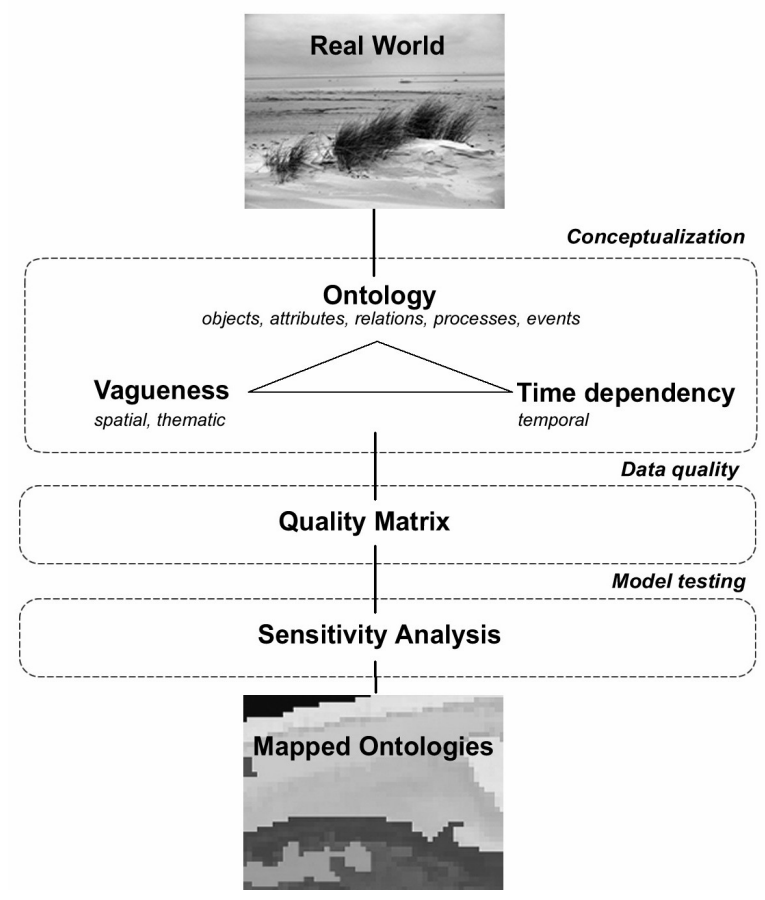

Figure 1. Modeling of geospatial dynamics, from features in the real world to objects in the model.

\subsection{Ontology}

A common reasoning framework clarifies the structure of knowledge, and leads to coherent knowledge base (Jeansoulin and Wilson, 2002). An ontology describes "the metaphysical study of the nature of being and existence" (Frank, 2003b). It describes a conceptualization of the real world and is closely related to software engineering activities like conceptual analysis and domain modeling (Guarino, 1998). An ontology determines what is independent of an observer. This includes physical reality, as the position of an object in Cartesian space, but also human agreements, e.g. classification rules or social arrangements. At the heart of Aristotle's ontology is a theory of 'substances' (things, or bodies) and 'accidents' (qualities, events, processes) (Smith, 2001).

We address management of dynamic landscape features in an ontological approach as an integration of data with semantics. For modeling dynamic landscape features, we propose a general ontology for objects in the real world, derived from monitoring activities. Objects are characterized by their 'substances' and 'accidents', whereby 'substances' are attributes $(a t t)$, which are dependent in space $(x, y)$, value $(v)$ and time $(t)$, and have relationships between other objects ( $r e l)$. 'Accidents' are related to the spatio-temporal behavior of the datasets, and include events (ev) and processes (proc). Hence, an ontology exists of objects, their attributes and relationships, events, processes and states (Chandrasekaran et al., 1999; Kuhn, 2001). For an application in landscape monitoring, we define

object $\in S_{o b j}\left(a t t_{\alpha}, \operatorname{proc}_{\beta}, e v_{\gamma}\right)$

where $S_{o b j}$ is the landscape object conceptualized by an onto$\operatorname{logy}$ and where $\alpha, \beta$ and $\gamma$ point to an index set.

Equation 1 shows that each particular object is characterized by a specific set of attributes, processes and events. For continuous attributes with index set $\alpha$ it follows that:

$a t t_{\alpha}=f_{\text {att }}(x, y, v, t)$

Equation 2 illustrates a field model, where each point in space and time for different properties can be observed. Ontological features (objects, attributes, relations, processes and events) specify the ontology for an application. Processes are time dependent and require a special set of attributes. The value of the process is not only dependent on the attribute value, but also on situations in the past, trend, expectations and random noise. Events are special processes, i.e. they occur suddenly and may change attribute values and processes.

\subsection{Implementation of Fuzzy Rules}

Landscape units are by nature vague in their content and extent (Frank 2003b; Fisher et al., 2005). For vague objects three types of uncertainty exists (Molenaar, 1998): 
1. Existential uncertainty expresses how sure we are that an object really exists.

2. Extensional uncertainty reflects the spatial extent of an object.

3. Geometric uncertainty refers to the precision with which the boundary of an object can be determined.

In this study we focus on the existential and extensional uncertainty. To do so, we implement fuzzy rules using membership functions to describe the spatial and thematic uncertainty of the variables that describe objects. These membership functions are based on semantic import models for each attribute, with a value 0 as no membership, a value 1 as full membership, and a value in between as partial membership. We adapt the field model for attributes from equation 2 to:

$a t t_{\alpha}=m v_{\alpha} \cdot f_{a t t}(x, y, v, t)$

For index set $\alpha$, a membership function $m v_{\alpha}$ is included to express the spatial $(x, y)$, thematic $(v)$ and temporal $(t)$ uncertainty of the attributes.

\subsection{Time Correction}

Landscapes can be observed in space, time and theme (Peuquet, 1994; Peuquet, 2002). Since dynamic landscape features can not be monitored in real time, but only in a representation of reality, we need to abstract temporal effects to create snapshots of the world. This can be described by the following equation, which Goodchild called 'geographical reality' (Goodchild, 1992):

$a t t=f_{\text {att }}(x, y, v)$

To analyze and understand dynamic landscape features we extend spatial ontologies for representing space and time (Claramunt, 1997; Frank, 2003a,b). By doing so, temporal inaccuracies should be accounted for. The temporal inaccuracy can affect the accuracy of time measurement of the data, the temporal validity of the data or the temporal consistency of the data.

This study manages temporal inaccuracies that occur due to temporal variability of the data. For any $\alpha$ at time $t$ we implement correction factors $\left(C F_{\alpha, t}\right)$ to correct for this temporal uncertainty, whereby $C F_{\alpha, t}$ defines the 'degree of certainty' for time $t$ of data capture, with values between 0.5 and 1 . This correction factor is applied on the basis of the slope of the fuzzy membership function. If $C F_{\alpha, t}=1$, the temporal certainty of the data capture is high, and the membership function will be identical as equation 3. If $C F_{\alpha, t}<1$, the temporal certainty of data capture is lower and the slope of the membership function will become less steep according to:

$a t t_{\alpha, t}=C F_{\alpha, t} \cdot m v_{\alpha, s l o p e} \cdot f_{a t t}(x, y, v)$
This correction factor only applies on the slopes of the membership functions, i.e. the location where there is no full membership or non-membership. Here, the transition zone described by partial memberships will therefore increase.

\subsection{Quality Matrix}

Fitness for use models the relation between available data and data required to analyze landscape phenomena (Hunter, 2001). Herein a quality matrix, where ISO quality elements (ISO, 2003) are portrayed against application features derived from the ontology, can be practical. Features and quality elements are defined first, followed by integration with ontological concepts. Each column of the quality matrix indicates an ontological feature, each row the quality elements. The relevant cells of the matrix link, when applicable, a quality value to a feature.

A quality matrix may assist a decision maker, for example, to find out the best available dataset for an application. It comprehensibly formalizes spatio-temporal problems, taking into account the objects, fuzzy rules and integration of temporal dimensions, and current standards of quality.

\subsection{Model Testing}

Establishing the usefulness of models as a means of improving our understanding of predicting geospatial dynamics requires use of objective measures of performance (Gardner and Urban, 2003). Testing occurs throughout stages of model development by means of measures on the model structure, parameter sensitivity, model adequacy and hypothesis testing. A quantitative model that exhibits large fluctuations in output for relatively small changes in the value of some input parameters is sensitive to the parameter, whereas a model which exhibits small output variations for substantial perturbations is insensitive to the parameter.

We test the beach nourishment model using a sensitivity analysis, which determines the model response to any realistic set of parameter perturbations (Rose and Swartzman, 1981). Therefore, we apply a probabilistic sensitivity analysis technique that reflects the likely value of an uncertain parameter, based on the probability distribution. We select bounds for a confidence interval for all possible values of the parameter.

\section{Beach Nourishment Application}

\subsection{Study Area and Dataset}

The study area is located at the north-western part of Ameland, a coastal barrier island on the fringe between the Wadden Sea and the North Sea (Figure 2). Geomorphological processes such as erosion, transport and sedimentation of sandy materials are causing major changes that have to be compensated by sand suppletion.

The dataset for this area consists of multi-temporal digital elevation models (1980-2003) and satellite imagery. Each digital elevation model is derived from the DONAR database (Eleveld, 1999). The DONAR database contains annual beach 
and foredune elevation transects. The transects are 200 to 250 $\mathrm{m}$ apart and elevation is measured at $5 \mathrm{~m}$ intervals along a cross-shore line. The underwater part of the profile is measured with echosoundings from ships with automatic positionfinding systems. The elevation measurements are executed by means of laser altimetry. From the point data, elevation data are interpolated towards a $30 \mathrm{~m} \times 30 \mathrm{~m}$ grid, using the IDW interpolator of ARCGIS geostatistical analyst. This grid size corresponds to satellite image pixel size, derived from Landsat5-TM and Landsat7-ETM+ satellites. The beach objects are thus structured into compartments within a higher conceptual level, based on perceivable regions on the beach. Compartments are the regions between two transects. For this study we take the dataset for 1995 as illustration for modeling beach nourishments.

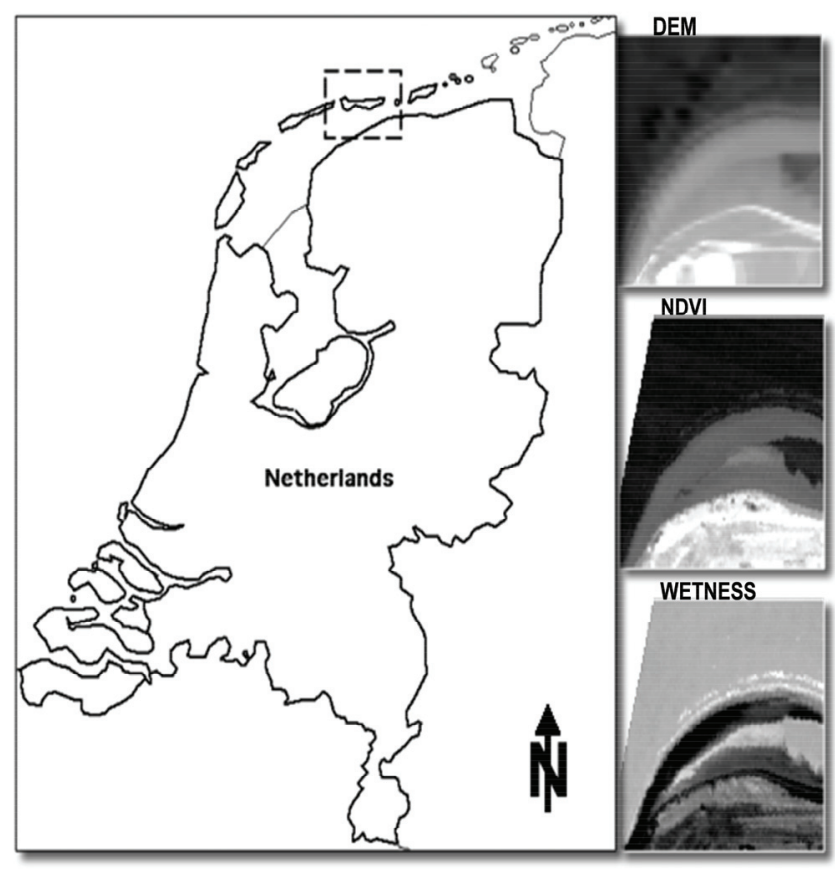

Notes: The study area is located in the northwestern part of Ameland The study area is depicted by a DEM, NDVI and Wetness Index derived from Landsat TM (1995).

Figure 2. The Isle of Ameland located in the north of the Netherlands.

\subsection{Beach Nourishment}

For beach management purposes, Rijkswaterstaat, the directorate-general of the Ministry of Public Works responsible for the maintenance of the coast, divides the beach area into compartments. Each compartment has two boundaries to its adjacent compartment $\left(C L_{1}\right.$ and $\left.C L_{2}\right)$, a beach-sea boundary $(B S)$ and a beach-dune boundary $(B D)$. Here, beach nourishments are carried out to address changes in morphology with consequences for the public safety. Beach areas suitable for nourishment are identified by localizing them and assessing their sizes. A decision maker is then assisted to manage nourishments in time.

Traditionally, beach nourishments are carried out by dump trucks restricted within these compartments. To localize and assess these beach areas, the required volume of sand need to be calculated, which requires determination of the expected erosion, the recurrence interval and the sand reserve. The sand reserve, i.e. the beach volume at time $(t)$ can be calculated from the digital elevation models. Reference is made to the basal coastline, being the coastline position on January $1^{\text {st }} 1990$. Beach nourishments are carried out when the beach volume at actual coastline is below the volume at basal coastline. The actual beach volume is calculated by multiplying the beach surface area with the elevation $(e)$. Elevation $(e)$ is the difference between the actual elevation measurement and the lower computing boundary, i.e. $-4.2 \mathrm{~m}$. at Dutch standard sea-level (= NAP) (Roelse, 2002).

So far, volumes of sand are calculated as crisp objects on the basis of these compartments. The interest of this application is to take into account the 'fuzzy' nature and the 'dynamics' of these objects. Besides, with the assistance of navigational systems, dump trucks can precisely nourish beach areas and are not restricted to compartment limits.

Identification of areas that require beach nourishment depends upon terrain elevation (height around zero), vegetation index (non-vegetated zones) and wetness index (dry zones). Elevations between -1.1 and $2 \mathrm{~m}$ at Dutch standard sea-level (NAP) are considered as beach areas. Such areas are derived from digital elevation models (DEMs). Similarly, non-vegetated and dry zones are derived from the Landsat TM imagery, using respectively Normalized Difference Vegetation Index (NDVI) (Rouse et al., 1974) and wetness index (Crist and Cicone, 1984). The NDVI is a spectral transformation that is applied to bands 3 and 4 of the Landsat image for the purpose of assessing the health and vigor of vegetated surfaces. NDVI has the advantage of simplicity and common use by remote sensing analysts (Dunham et al., 2005). The wetness index is a tasseled cap transformation that provides excellent information for land use applications because it allows the separation of barren (bright) soils from vegetated and wet soils. Non-vegetated zones are selected as areas with negative NDVI values; dry zones are selected as areas with a wetness index lower than zero. The delineation of the object beach should satisfy the constraints for elevation, non-vegetated and dry zones, e.g. the so-called beachplain (Van de Vlag et al., 2005).

The structural erosion per compartment is determined, by plotting the beach volumes from before 1990 against the beach volume with the basal coastline. A negative trendline indicates erosion, whereas a positive trendline indicates sedimentation.

Two constraints apply when deciding upon nourishment; $\mathrm{C}_{1}$ : a coast compartment shows structural erosion, $\mathrm{C}_{2}$ : the volume for beach nourishment should exceed $200,000 \mathrm{~m}^{3}$. Constraint $\mathrm{C}_{2}$ is a soft constraint, as nourishment may be carried out, depending on local and regional policies. 


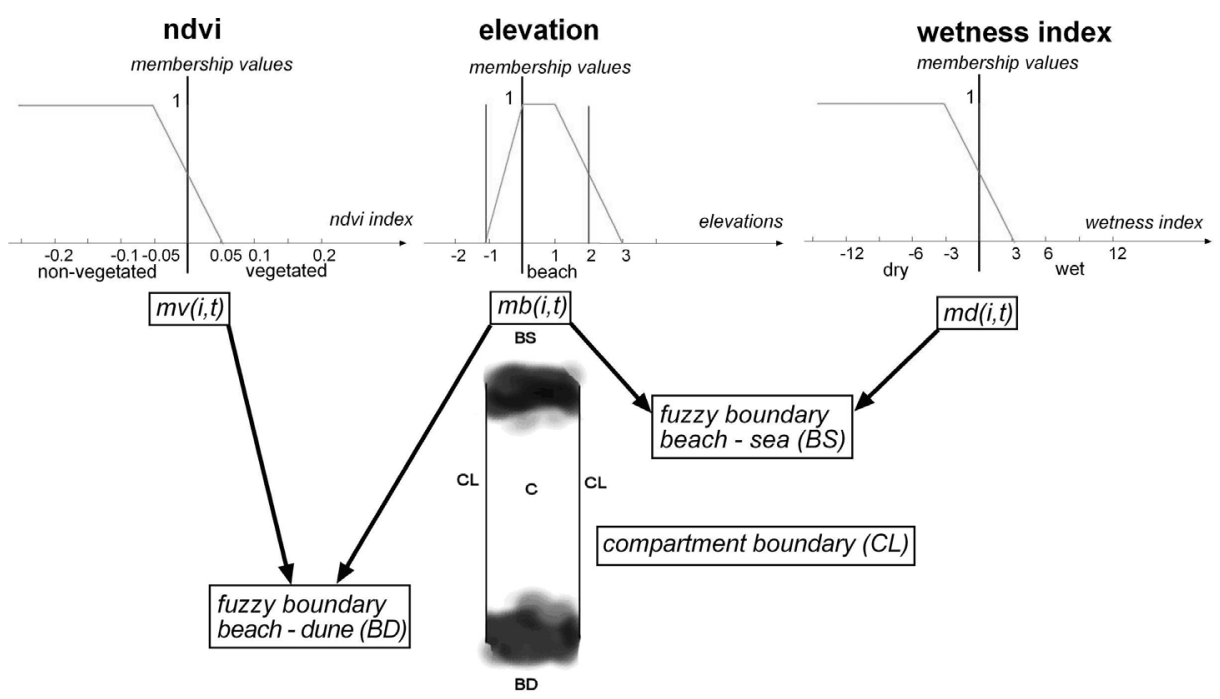

Notes: The lower image visualizes a compartment $(C)$, with two adjacent crisp boundaries $(C L)$ and two fuzzy boundaries $(B S)$ and $(B D)$.

Figure 3. Compartment, boundaries and their various fuzzy membership functions.

\subsection{Modeling Beach Nourishment}

Beach nourishment is modeled in three different ways. Firstly, a crisp approach considers sharp boundaries between different beach objects corresponding to a traditional approach. Secondly, a fuzzy approach describes compartments as the membership functions of three parameters. Thirdly, temporal membership functions are included for modeling temporal processes.

\subsubsection{Crisp compartmental method (CC)}

Identification of beach areas that require beach nourishment requires compartments $(C)$ to distinguish zones with sedimentation from those with erosion. These compartments are perpendicular to the coast, 100 to $200 \mathrm{~m}$ wide, and fixed in time. Compartment width is a fixed limit (CL.geo). Compartment length on the other hand, being the distance between the beach-sea boundary (BS.geo) and the beach-dune boundary $(B D . g e o)$, is fuzzy, as both boundaries are dynamic (BS.geo $(t)$, $B D . g e o(t))$, due to erosion and sedimentation. For the crisp compartmental (CC) approach, these boundaries are assumed to be sharp.

On the basis of trendline calculation the decision-maker decides if sedimentation and erosion occur within a compartment. On account of beach volumes, the amount of erosion and sedimentation is calculated for $n p$ pixels each of size $p s$. The beach volume within a compartment $(C \cdot \operatorname{vol}(t))$ is calculated as:

C.vol $(t)=p s^{2} \times \sum_{i=1}^{n p} e(i, t)$

where $e(i, t)$ is the elevation of pixel $i$ at time $t$. A compartment is indicated for nourishment, when the beach volume in a compartment shows structural erosion, e.g. obeying the two constraints $\left(\mathrm{C}_{1}\right.$ and $\left.\mathrm{C}_{2}\right)$.

\subsubsection{Fuzzy compartmental method (FC)}

Spatial and thematic uncertainty of the attributes are modeled using fuzzy logic. The beach compartments suitable for nourishment are identified by their memberships to dry nonvegetated beaches. Hence, a compartment is bound by two static compartment boundaries (CL.geo) and by two fuzzy boundaries: the sea-beach boundary $(B S . g e o(t))$ and the beachdune boundary $(B D . g e o(t))$. These boundaries are illustrated in Figure 3.

The sand volume within the fuzzy compartmental (FC) method can be calculated, using:

$$
\operatorname{C.vol}(t)=p s \times \sum_{i=1}^{n p} m(i, t) \times e(i, t)
$$

where $m(i, t)$ equals the membership value of location $(i)$ in compartment $C$ at time $t$. It is calculated as:

$m(i, t)=\min \{m b(i, t), m d(i, t), m v(i, t)\}$

where $m b(i, t)$ is the membership function of the beach object, $m d(i, t)$ that of dry object and $m v(i, t)$ that of a non-vegetated object in which pixel $i$ occurs at time $t$. Membership functions are compiled as triangular functions. The $m b(i, t)$ equals 1 if elevation ranges from 0 to $1 \mathrm{~m}$ amsl, it increases linearly from 0 to 1 between -1.1 to $0 \mathrm{~m}$ amsl and decreases linearly from 1 
to 0 between 1 and $3 \mathrm{~m}$ amsl, and it equals 0 elsewhere. The soil is wet if the wetness index is positive, and dry if it is negative. A membership function $m d(i, t)$ for the fuzzification equals 1 if wetness index is less than -3 , and decrease linearly from 1 to 0 for the wetness index moving from -3 to $3 \mathrm{~m}$, and it equals 0 elsewhere. Land is covered with vegetation if the NDVI is positive and is bare if it is negative. A membership function $m v(i, t)$ equals 1 if the NDVI value is less than -0.05 , it equals 0 if the NDVI is larger than 0.05 and it decrease linearly from 1 to 0 in between.

\subsubsection{Temporal fuzzy compartmental method (TFC)}

To include temporal uncertainty into the beach nourishment processes, the daily fluctuations for the wetness index, the monthly fluctuations for the vegetation index and yearly fluctuations for the elevation are considered. Three temporal membership functions are introduced to reflect the appropriate time scale for these attributes. In the Netherlands, the growing season for vegetation starts early March and reaches maximal values in June. Hence, in June the certainty to detect healthy fully-grown vegetation and thus large NDVI values is high. The temporal membership function $n v(t)$ corresponds to the growing season and equals 1 between 1 June and 1 August, it equals 0.5 between 1 November until 1 March, and it is linear in between. Similarly for soil wetness, the temporal membership function $n d(t)$ corresponds to tide fluctuations and equals 1 during flood time and equals 0.5 during low tide, and further follows a sine form, i.e. $n d(t)=0.75+0.25 \cdot \cos (2 \pi \cdot t / 12.5)$, with $t$ expressed in hours in relation to high tide. Finally for elevation, the temporal membership function $n b(t)$ is constant and describes the actual digital elevation model.

The temporal fuzzy approach (TFC) first calculated correction factors $(C F \mathrm{~s})$ derived from introducing date and time of data capture into the temporal membership functions. Next, the spatial membership functions originate from fuzzy compartmental method $m b(i, t), m d(i, t)$ and $m v(i, t)$ are corrected with this correction factor (Figure 4). Low $C F$ s lead to membership functions that are less steep; $C F$ s close to 1 result in membership functions similar to those in $m b(i, t), m d(i, t)$ and $m v(i, t)$.

FC and TFC differ from each other, as the slope of the membership function is corrected according to the temporal (un)certainty of the vegetation- and wetness-index. At high tide, the certainty that a beach is wet or dry is high, while at low tide this temporal certainty is low. Similarly, in summer the certainty about vegetation is high, while during growing season this certainty is lower. For 1995, the capturing date of the Landsat image is 7 November, at $9 \mathrm{~h}-30$, just after low tide corresponding with a low temporal certainty for vegetation and wetness. The image is radiometric corrected to correct for atmospheric conditions that may affect NDVI and wetness index values.

\subsection{Quality Elements and Quality Matrix}

Before constructing a quality matrix, spatial data quality elements need to be defined for the beach nourishment application. For spatial uncertainty, the following ISO quality elements are considered important (ISO 2003):

1. Positional accuracy, whereby sub-elements of interest are: 1) relative or internal positional accuracy, i.e. closeness of the relative positions of objects in a dataset to their respective relative positions accepted as or being true; 2) gridded data position, i.e. closeness of gridded data position values to values accepted as or being true.

2. Thematic accuracy, with sub-elements of interest: 1) the accuracy of quantitative attributes, i.e. the correctness of quantitative attributes and of the classifications of objects and their relationships; 2) classification correctness, i.e. comparison of the classes assigned to the objects or their attributes to a universe of discourse (e.g. ground truth or reference dataset).

3. Temporal uncertainty of the compartments can be recognized by temporal accuracy. In particular the sub-element for accuracy of a time measurement, i.e. correctness of the temporal references of an item (reporting of error in time measurement).

4. Completeness; this is determined by sub-elemental data completeness, i.e. the commission and omission of datasets.

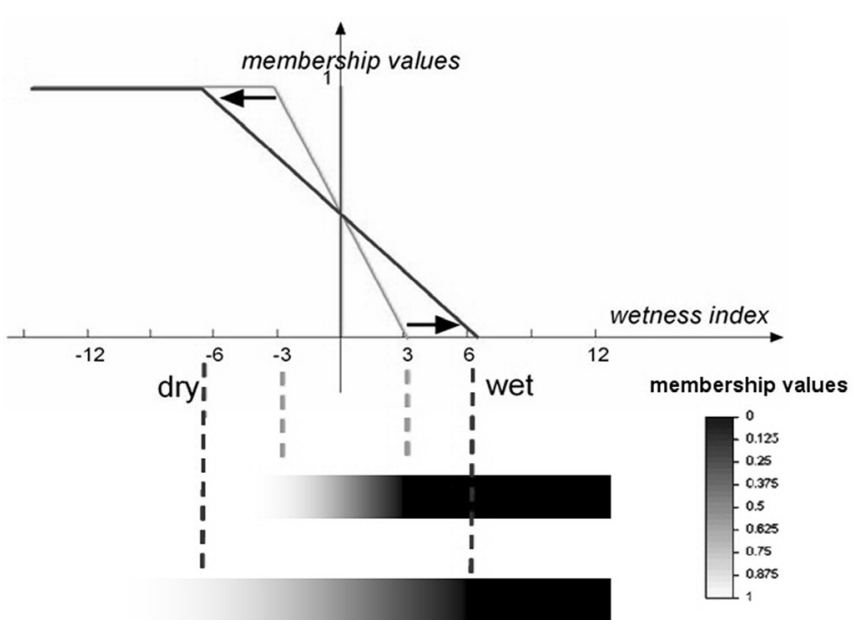

Figure 4. The slope of the membership function for dry beach areas (corrected for tide influences; the vagueness of the boundary area will increase).

By applying an ontological approach, a quality matrix is constructed, projecting ontological features such as objects, attributes, relationships, processes and events against the ISO quality elements.

\subsection{Sensitivity Analysis}

By means of a sensitivity analysis we can determine the effects of the spatial and temporal influences of attributes to the amount of beach nourishment. The sensitivity of calcu- 


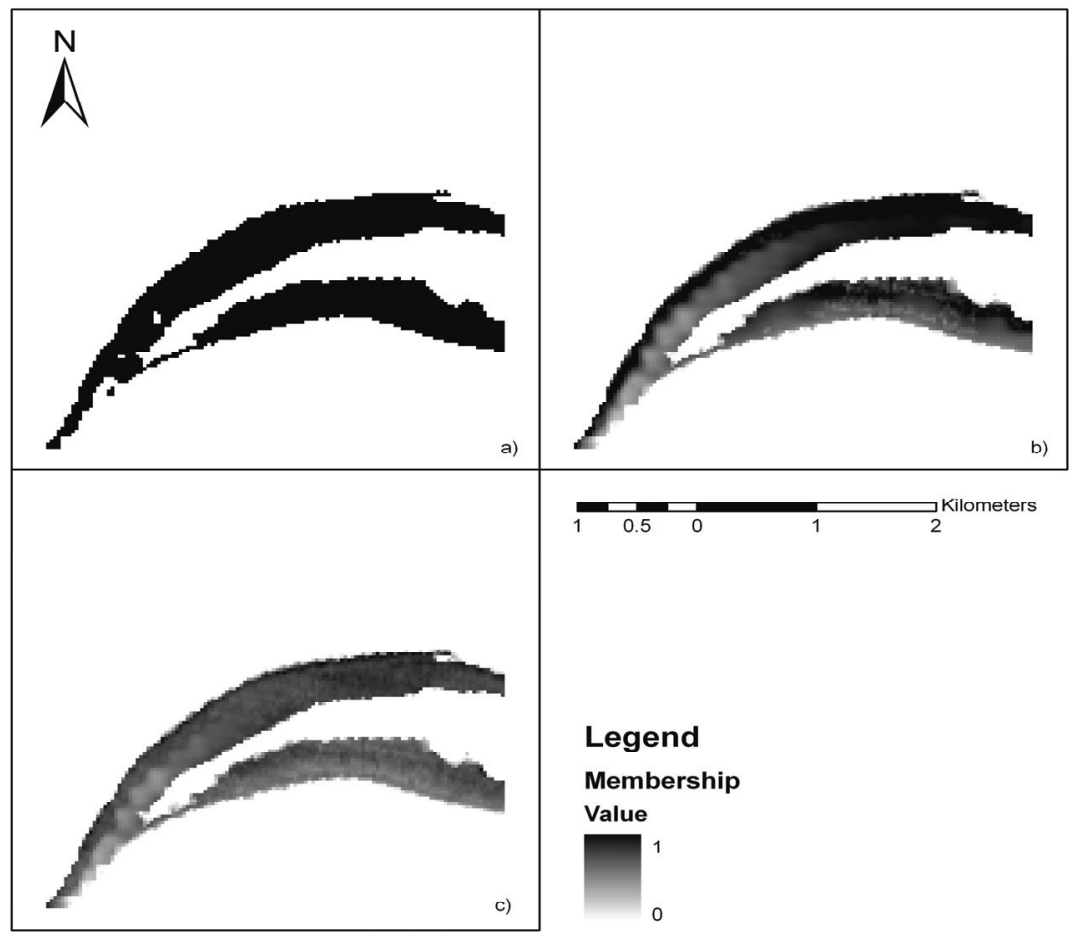

Figure 5. Beachplain classification, using a crisp (top left), fuzzy (top right) and temporal fuzzy (bottom) method.

lated beach nourishment volumes is based on statistical properties, i.e. the variance of the attribute elevation, NDVI and wetness. We also investigate the sensitivity of the output on statistical properties of the tide cycle and vegetation growth.

For NDVI and wetness, sensitivity analysis has been executed with 1 standard deviation range from the dividing value 0 . A positive NDVI value corresponds, as at least theoretically, with presence of vegetation and a negative value with absence of vegetation, whereas positive and negative wetness values correspond to wet and dry, respectively.

For elevation, we observed the cross-validated RMSE by interpolating the elevation profiles to grid maps. Here, $5 \%$ of the data points are used for cross validation, to obtain an accuracy value of $Z$ value. For 1995 , the cross-validated RMSE is $0.28 \mathrm{~m}$ for half width of $95 \%$ confidence interval. The sensitivity analysis for elevation is carried out within the upper and lower limit of the cross-validated RMSE for the interpolated $Z$ values, i.e. from $Z-0.28 \mathrm{~m}$ as the lower limit to $Z+$ $0.28 \mathrm{~m}$ as the upper limit.

For the TFC method choices in the correction factor $(C F)$ of the temporal membership functions were evaluated. These choices represent correction factors values that are in the vicinity of our choices, with minimum of 0.5 and maximum of 1 . A value equal to 0 would be highly unlikely. Therefore, the correction factor $(C F)$ in the membership function for the tidal cycles is varied between 0.3 and 0.7 , whereas its highest value is kept equal to 1 when we observe perfect timing of data capture. Also, the correction factor in the membership function for the vegetation growth cycle varies between 0.3 and 0.7 , with the highest value equal to 1 .

\section{Results}

\subsection{Comparison of the Approaches}

The methodology used in this study construct in three phases a spatio-temporal ontology for the beach nourishment application. The CC defines beach areas suitable for nourishment by crisp boundaries of the compartment. The object of interest is the compartment $(C . i d)$, whereby the sand volume calculation $(C . v o l)$ is grounded on the minimum intersection of the attributes elevation, vegetation and wetness. The FC defines the beach areas suitable for nourishments by fuzzy boundaries of the compartment. The sand volume calculation is then based on the minimum intersection of the membership functions of the attributes elevation, vegetation and wetness. For the TFC, the sand volume calculation is corrected for temporal uncertainty and is the minimum intersection of the corrected membership functions of the attributes.

Results are presented in Figure 5, showing the beachplain as the beach area that is dry and non-vegetated. The slope of the membership functions for vegetation and wetness is corrected for temporal uncertainty, and is less steep, resulting in a fuzzy beach plain (Figure 5b,c).

After trendline calculation, two regions are indicated as 


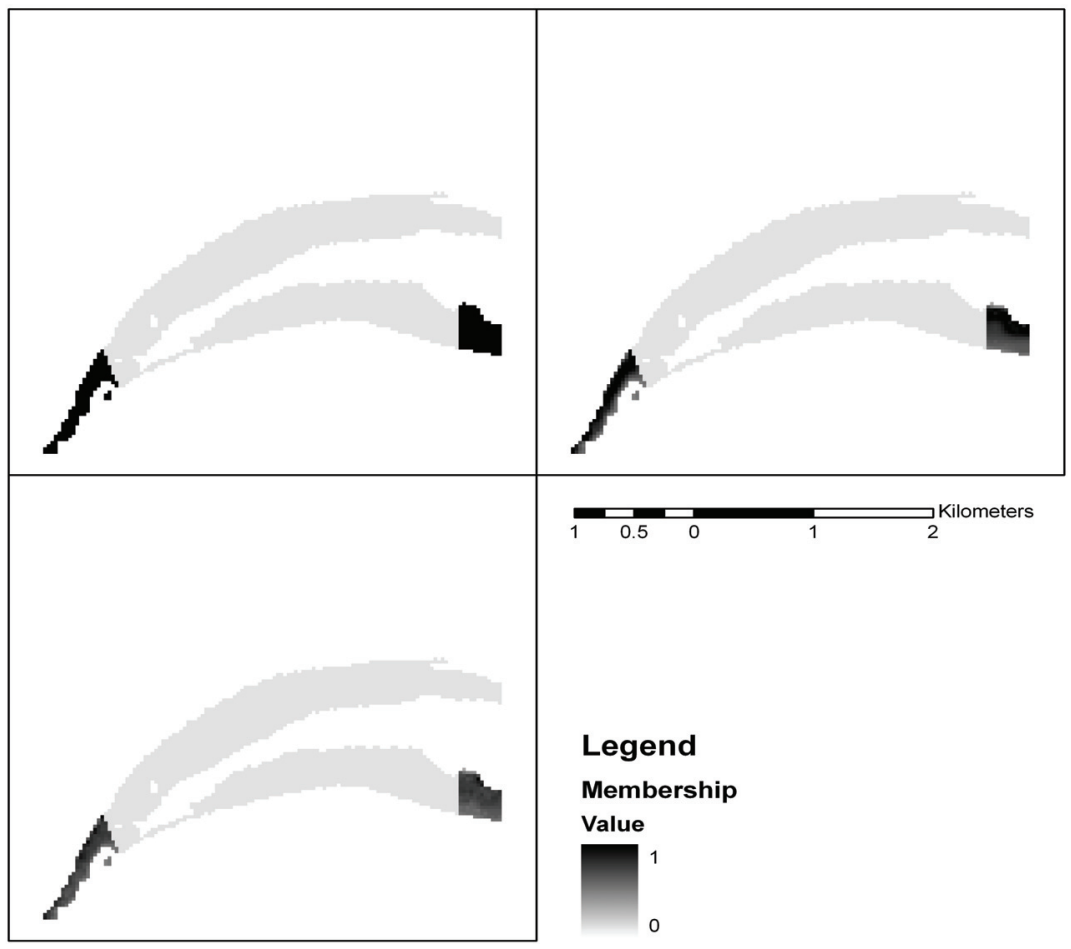

Figure 6. The final result of CC (top left), FC (top right) and TFC (bottom).

areas with structural erosion (Figure 6). The southwest region is of interest, as most actual beach nourishments are actively carried out in that area. Moreover, the threat for safety for the public is higher, as there is only a single row of dunes to protect the hinterland. The calculation for beach volumes, using the compartmental methods, is represented in Table 1. The fuzzy approach indicates lower volumes, as thematic uncertainty, as well as temporal uncertainty is accounted for. The volumes in Table 1 are below the criteria for nourishment $\left(200,000 \mathrm{~m}^{3}\right)$, and beach nourishment in 1995 will not be carried out.

Table 1. Beach Nourishment Volumes Using CC, FC and TFC

\begin{tabular}{cccc}
\hline & $\mathrm{CC}$ & $\mathrm{FC}$ & $\mathrm{TFC}$ \\
\hline Volume $\left(\mathrm{m}^{3}\right)$ & 78,600 & 58,700 & 51,600 \\
\hline
\end{tabular}

\subsection{Quality Elements}

Table 2 describes the quality of objects and attributes that applies to the case study. The objects consist of compartment $(C . i d)$ and its boundaries (CL.id, BD.id, BS.id). The attributes consider elevation (BD.z, BS.z), wetness index (BS.wi), vegetation index (BD.ndvi) and structural erosion (C.se, C.vol90). The prominent feature of interest is the amount of beach volume, represented by C.vol in Table 2. Row wise, the quality elements are described. Positional accuracy is represented by relative and gridded data position. Different membership functions related to corresponding attributes occur for classification correctness and temporal accuracy. The quantitative attribute accuracy is only valid for the attributes considering elevation for 1995 (BD.z, BS.z, C.vol). The data completeness is illustrated in the last column and equals $86.7 \%$ for the 1995 data set.

We note that the three different procedures lead to different quality assessments. The $\mathrm{CC}$ results in statements on objects that have a positional accuracy. The FC describes both the positional and the thematic accuracy. Thematic accuracy is described by membership functions that were included from the semantic import model. Finally, the TFC includes statements on positional, thematic and temporal aspects. Note that the temporal membership functions were also based on a semantic import model (see section 3.3.3.).

\subsection{Sensitivity Analysis}

Beach nourishment volumes are affected by choices for elevation (Figure 7a). Generally, an increase is observed with an increasing elevation, which is most prominent for $\mathrm{CC}$, and less prominent for $\mathrm{FC}$ and $\mathrm{TFC}$, with values ranging from 30,000 to $100,000 \mathrm{~m}^{3}$. Hence, interpolation uncertainties may have a large influence on the calculated beach volumes. Similarly, the volumes are sensitive for the choice of the dividing NDVI value, with a sharp jump around the NDVI value of 0 (Figure $7 \mathrm{~b}$ ). We notice that FC and TFC give almost similar 

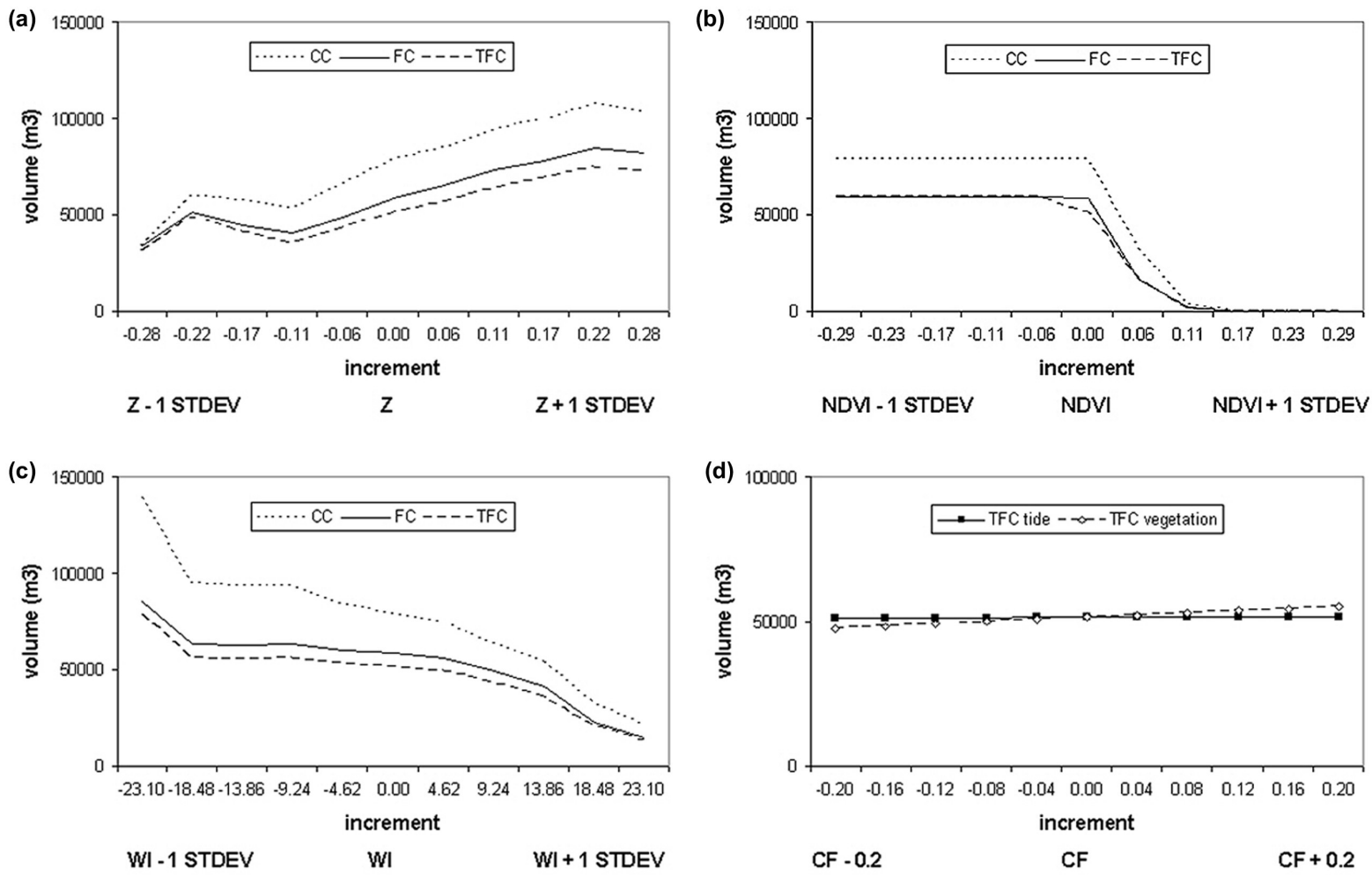

Figure 7. The results of sensitivity analysis using CC, FC and IFC for (a) elevation, (b) NDVI and (c) wetness index; for temporal variables (d) only the TFC is applied.

Table 2. Quality Elements for the Ontological Features for 1995

\begin{tabular}{lllllll}
\hline \multirow{2}{*}{ Objects } & \multicolumn{2}{l}{ Positional Accuracy } & \multicolumn{2}{l}{ Thematic Accuracy } & \multicolumn{1}{l}{ Temporal Accuracy } & Completeness \\
\cline { 2 - 6 } & Rel. & Grid. & ClasCor & QAA & ATM & Data \\
C.id & $48.6 \mathrm{~m}$ & $30.3 \mathrm{~m}$ & & $<1$ year & $86.7 \%$ \\
CL.id & $\mathrm{NR}$ & $30.3 \mathrm{~m}$ & & $<1$ year & $86.7 \%$ \\
BD.id & $48.6 \mathrm{~m}$ & $30.3 \mathrm{~m}$ & & $<1$ year & $86.7 \%$ \\
BS.id & $48.6 \mathrm{~m}$ & $30.3 \mathrm{~m}$ & & & $<1$ year & $86.7 \%$ \\
Attributes & & & & & \\
C.vol & $48.6 \mathrm{~m}$ & $30.3 \mathrm{~m}$ & $\mathrm{~m}(\mathrm{i}, \mathrm{t})$ & $\pm 0.28 \mathrm{~m}$ & $\mathrm{CF} \bullet \mathrm{m}(\mathrm{i}, \mathrm{t})$ & $86.7 \%$ \\
C.vol90 & $48.6 \mathrm{~m}$ & $30.3 \mathrm{~m}$ & $\mathrm{~m}(\mathrm{i}, \mathrm{t})$ & $\mathrm{NR}$ & $\mathrm{CF} \bullet \mathrm{m}(\mathrm{i}, \mathrm{t})$ & $\mathrm{NR}$ \\
BD.ndvi & $48.6 \mathrm{~m}$ & $30.3 \mathrm{~m}$ & $\mathrm{mv}(\mathrm{i}, \mathrm{t})$ & $\mathrm{NR}$ & $\mathrm{nv}(\mathrm{t})$ & $86.7 \%$ \\
BD.z & $\mathrm{NR}$ & $30.3 \mathrm{~m}$ & $\mathrm{mb}(\mathrm{i}, \mathrm{t})$ & $\pm 0.28 \mathrm{~m}$ & $\mathrm{nb}(\mathrm{t})$ & $86.7 \%$ \\
BS.wi & $48.6 \mathrm{~m}$ & $30.3 \mathrm{~m}$ & $\mathrm{md}(\mathrm{i}, \mathrm{t})$ & $\mathrm{NR}$ & $\mathrm{nd}(\mathrm{t})$ & $86.7 \%$ \\
BS.z & $\mathrm{NR}$ & $30.3 \mathrm{~m}$ & $\mathrm{mb}(\mathrm{i}, \mathrm{t})$ & $\pm 0.28 \mathrm{~m}$ & $\mathrm{nb}(\mathrm{t})$ & $86.7 \%$ \\
C.se & $48.6 \mathrm{~m}$ & $30.3 \mathrm{~m}$ & $\mathrm{~m}(\mathrm{i}, \mathrm{t})$ & $\mathrm{NR}$ & $\mathrm{CF} \bullet \mathrm{m}(\mathrm{i}, \mathrm{t})$ & $86.7 \%$ \\
$\ldots$ & $\ldots$ & $\ldots$ & $\ldots$ & $\ldots$ & $\ldots$ & $\ldots$ \\
\hline
\end{tabular}

*Abbreviations: ClasCor = classification correctness, QAA = quantitative attribute accuracy, ATM = accuracy of time measurement, $\mathrm{NR}=$ not relevant 
results, whereas the jump around the NDVI value of 0 for $\mathrm{CC}$ is even more prominent. An explanation is that a small change in positive NDVI values signifies pioneering vegetation as vegetation, decreasing the sand volumes for beach nourishments. Also, the standard deviation is relatively large, as NDVI values equal to 0 for beach and sea, and have positive values for dunes. For wetness we notice a dependence on the choice of equilibrium (i.e. wetness index equals 0 ), with a decrease of the volume of beach nourishment with increasing wetness value (Figure 7c).

Interestingly, the large sensitivity of the results obtained for the spatial variables does not lead to modifying assumptions made in the space-time membership function. The volume of beach nourishment shows changes in the range of 51,400 to $51,700 \mathrm{~m}^{3}$ for modifying assumptions on the tidal membership function, and values from 47,200 to $55,100 \mathrm{~m}^{3}$ for the assumptions in the vegetation growth cycle (Figure 7d). It demonstrates that the model is rather insensitive to these assumptions.

\section{Discussions}

In this study we used an ontological approach to describe beach objects for nourishment. This approach concerns space, theme and time issues for a dynamic geographic application. The use of identical ontologies over time can help to detect and determine objects that change between 'snapshots'. On the one hand it gives a good understanding of the underlying spatio-temporal problem, i.e. the nourishment of beach areas in space and time; on the other hand it does not deal with different conceptual levels. With higher resolutions different attributes, processes and events to describe beach objects might be involved, leading to different spatio-temporal ontologies. Also, the selection of quality elements depends upon the spatial scale of the dataset.

The selection of quality elements essential for the study area is done by using previous studies (Cheng et al., 2001; Van de Vlag et al., 2005). The accuracy to describe objects requiring beach nourishment is important. Positional elements concern elevation, and a choice for boundaries between adjacent objects. The elevation measurements from the DONAR database are accurate and precise and therefore affect positional accuracy to a lesser extent. Positional accuracy is in particular determined by the grid resolution of the DEM and the geometric correction of the satellite imagery.

Thematic accuracy concerns determination of a classification using quantitative figures. We have chosen to apply semantic import models for implementation of fuzzy rules and these are based on expert knowledge, therefore this may be subjective and influenced by spatial variation.

In our study, we model temporal accuracy by seasonal factors for vegetation, by tidal fluctuations for the wetness index, and by both erosion/sedimentation processes and by incidental changes for elevation. Seasonal factors may be difficult to relate to vegetation, as other factors, such as moisture content and vegetation stress, contribute as well. Also relations between tidal effects and wetness are subject to factors like vegetation and weather. Currently, we did not consider weather influences as these are complicated to observe and difficult to model due to several time dimensions, e.g. influences like large storm events or wind erosion are measured on different temporal scales. Finally, processes on sedimentation and erosion are applicable to larger areas of land and events like severe drought and large storms may interfere.

Causes of uncertainty in objects to determine suitability for beach nourishment lie in natural variation of the object describing variables. Together with a poor definition of context and definition rules for nourishment, this requires objects to be treated as vague objects. Membership functions represent their thematic and temporal uncertainty. In this study, temporal uncertainty is treated as a part of thematic uncertainty and their interrelationship is joined inextricable. This is expressed by using temporal (un)certainty by correction of the transition zones of the membership functions for thematic uncertainty.

A realistic geospatial model to fully describe beach features may be complicated. Relationships between ontological features occur at multiple conceptual levels. Quality elements for each of these features contribute to the overall quality of such a model. Therefore, a hierarchical structure may lead to an improved description of ontological features, as well as its quality elements. Scale issues, in time and space, leads to a different conceptualization of a spatio-temporal problem and therefore a different ontology and quality elements. A hierarchical representation of a model, from a knowledge based or Bayesian perspective, can assist a decision maker in selecting the best dataset for the ontology and quality elements in mind.

The sensitivity analysis as carried out in this study highlights the effects of choices for spatial variables, and much less so for choices in the temporal membership functions. It appears, that at least for this study, temporal effects can relatively simply be modeled, and that any subjective choice has much less influence than choices in spatial values.

The approach used in this article can be applied to other spatio-temporal datasets, like monitoring areas sensitivity for bushfires. Here, the ontology considers several datasets for conceptualization, whereby these areas are described by weather conditions, flame characteristics, fuel characteristics, elevation, etc. All these attributes concerns their own space, theme and time issues, and have their influence on the quality of the dataset.

In a recent study about the history of coastal protection, Charlier et al. (2005) state that beach nourishments is still favored with many instances. The decision makers prefer commonly profile feeding by simple deposition of material on the beach. Beach nourishments may act as both protection and restoration projects and has been implemented in all continents. Accordingly, many lessons have been learned that encompass decision choices of feeding material, preservation of source material sites, wave climate, etc. Several researchers in the Netherlands examined these decision choices. Van Rijn (1997) studied the sediment transport and budget along the 
central coastal zone of the Netherlands. He developed a mathematical model to calculate sand volume changes, based on hydrodynamic (waves and currents) and sand transport processes. Van der Wal (2000) examined the side-effect of beach nourishments on the rate of aeolian sand transport in the foredune area of central Ameland and concluded that grain size and adaptation length, which is a measure of the distance over which the sediment transport will adapt to a new equilibrium condition, both affected the topography of the beach-foredune area. Van Noortwijk and Peerbolte (2000) and Van Vuren et al. (2004) have studied extensively the optimal economical aspects with respect to the recurrence interval of beach nourishments in the Netherlands. In our approach, the ontologies are grounded on geographical choices to identify beach objects suitable for nourishment. The categorization of these objects, as well as the implementation of fuzzy set techniques and landscape dynamics, precedes the beach nourishment process.

\section{Conclusions}

This study exploits the use of spatio-temporal ontologies for describing beach objects suitable for beach nourishments. The spatio-temporal ontology includes fuzziness to describe vagueness of landscape features. The fuzzy approach is more realistic, as the described objects are more similar to those occurring in decision procedures and to the use in ontologies. The spatio-temporal ontology also includes important temporal aspects by means of temporal membership functions. Hence, the spatio-temporal ontology is therefore more generally applicable to space-time studies.

The case study focuses on beach nourishment at the Isle of Ameland. It is shown that the three methods can be implemented and applied to determine the required amount. Three approaches resulted in different amounts of beach nourishment volumes, ranging from 51,600 to $78,600 \mathrm{~m}^{3}$. By means of a quality matrix, the ontological features (i.e. objects, attributes, relationships) are projected against their quality elements. Further steps are identified to quantitatively evaluate different quality elements, such as positional, temporal and thematic accuracy. A sensitivity analysis showed that interpolation uncertainty for elevation and choices in dividing values between absence and presence of vegetation and between wet and dry soils may have a large influence on the calculated beach volumes. The influence of choices for the temporal membership function however is weaker.

Acknowledgments. The datasets have been made available by Rijkswaterstaat and RIKZ. The work was funded by the European Community, under IST-1999-14189 project REV!GIS. The authors acknowledge Bérengère Vasseur for the collaborative research and exchange of ideas.

\section{References}

Chandrasekan, B., Josephson, J.R. and Benjamins, V.R. (1999). What are Ontologies, and why do we need them? IEEE Intell. Syst., 14(1), 20-26.
Charlier, R.H., Chaineux, M.C.P. and Morcos, S. (2005). Panorama of the history of coastal protection. J. Coast. Res., 21(1), pp 79-111.

Cheng T., Molenaar M. and Hui L. (2001). Formalizing fuzzy objects from uncertain classification results. Int. J. Geogr. Inform. Sci., 15(1), 27-42.

Crist, H.P. and Cicone R.C. (1984). Application of the Tasseled Cap Concept to Simulated Thematic Mapper Data. Photogramm. Eng. Remote Sens., 50(3), 343-352.

Dunham, S., Fonstad, M.A., Anderson, S.A., Czajkowski, K., (2005). Using multi-temporal satellite imagery to monitor the response of vegetation to drought in the Great Lakes region. GIScience Remote Sens., 42(3), 185-201.

Eleveld, M.A. (1999). Exploring Coastal Morphodynamics of Ameland (the Netherlands) with Remote Sensing Monitoring Techniques and Dynamic Modeling in GIS, Ph.D. Dissertation, Amsterdam, The Netherlands, pp. 225.

Fisher, P. F., Wood, J. and Cheng, T. (2005). Fuzziness and ambiguity in multi-scale analysis of landscape morphometry, in F.E. Petry., V.B. Robinson and M.A. Cobb (Eds.), Fuzzy Modeling with Spatial Information for Geographic Problems, Springer-Verlag, Berlijn, pp. 209-232.

Foody, G.M. and Atkinson, P.M. (2002). Current status of uncertainty issues in remote sensing and GIS, in G.M. Foody and P.M. Atkinson (Eds.), Uncertainty in Remote Sensing and GIS, John Wiley \& Sons, New York.

Frank, A.U. (2003a). A linguistically justified proposal for a spatiotemporal ontology, COSIT 2003 conference. http://www.co mp.l eeds.ac.uk/brandon/cosit03ontology/position_papers/Frank.doc.

Frank, A.U. (2003b). Ontology for spatio-temporal databases, in $S p$ atio-Temporal Databases: The Chorochronos Approach, Lecture Notes in Computer Science, Berlin, Springer-Verlag, pp. 10-79.

Frank, A.U. and Grum, E. (2004). Proc. of the ISSDQ '04 Volume 1 and 2, 28a and b, GeoInfo Yellow Series, Vienna, Department of Geoinformation and Cartography, pp.369.

Gardner, R.H. and Urban, D.L. (2003). Model validation and testing: Past lessons, present concerns, future prospects, in C.D. Canham, J.C. Cole and W.K. Lauenroth (Eds.), Models in Ecosystem Science, Princeton University Press, Princeton, NJ, pp. 456.

Goodchild, M. (1992). Geographical data modeling. Comput. Geosci., 18(4), 401-408.

Gruber, T.R. (1993). A translation approach to portable ontology specifications. Knowl. Acquisition, 5 (2), 199-220.

Guarino, N. (1998). Formal ontology and information systems, in N. Guarino (Ed.), Proc. of FOIS'98, Formal Ontology and Information Systems, Trento, Italy, IOS Press, Amsterdam, pp. 3-15.

Hunter, G.J. (2001). Spatial Data Quality Revisited, in Proc. of GeoInfo 2001, Rio de Janeiro, Brazil, pp. 1-7.

ISO (2003). ISO/TC 211 Geographic Information/Geomatics-19113, 1914, Geographic Information Quality, and Evaluation Procedure, as sent to the ISO Central Secretariat for publication, pp. 68.

Jeansoulin, R. and Wilson, N. (2002). Quality of Geographic Information: Ontological approach and Artificial Intelligence Tools in the REV! GIS project, 8th EC-GI\&GIS Workshop, Dublin.

Kokla, M. and Kavouras, M. (2001). Fusion of Top-level and Geographic Domain Ontologies Based on Context Formation and Complementarily. Int. J. Geogr. Inform. Sci., 15(7), 679-687.

Kuhn, W. (2001). Ontologies in Support of Activities in Geographical Space. Int. J. Geogr. Inform. Sci., 15(7), 613-631.

Marceau, D.J., Guindon, L., Bruel, M. and Marois, C. (2001). Building temporal topology in a GIS database to study the land-use changes in a rural-urban environment. The Prof. Geogr., 53(4), 546-558.

Peuquet, D.J. (1994). It's about time: A conceptual framework for the representation of spatio-temporal dynamics in geographic information systems. Ann. Assoc. Am. Geogr., 84, 441-461. 
Peuquet, D.J. (2002). Representations of Space and Time, The Guilford Press, New York, pp. 380.

Roelse, P. (2002). Water en Zand in Balans, Evaluatie zandsuppleties na 1990, een morfologische beschouwing, Internal Report Rijksinsituut voor Kust en Zee, 2002.003, Middelburg, The Netherlands, pp. 108.

Rose, K.A. and Swartzman, G.L. (1981). A Review of Parameter Sensitivity Methods Applicable to Ecosystem Models, NUREG/ CR2016, Washington DC, US Buclear Regulatory Commission.

Rouse, J.W.Jr., Haas, R.H., Deering, D.W., Schell, J.A. and Harlan, J.C. (1974). Monitoring the Vernal Advancement and Retrogradation (Green Wave Effect) of Natural Vegetation, NASA/GSFC type III final report, Greenbelt, Maryland, NASA, pp. 371.

Shi W., Goodchild M.F. and Fisher P.F. (2003). Proc. of the Second International Symposium on Spatial Data Quality, Hong Kong Polytechnic University, Hong Kong, pp. 536.

Smith, B. (2001). Objects and their environments: From aristotle to ecological psychology, in A. Frank (Ed.), The Life and Motion of Socioeconomic Units (GISDATA 8), Taylor and Francis, London, pp. 79-97.

Van de Vlag, D.E., Vasseur, B., Stein, A., Jeansoulin, R. (2005). An application of problem and product Ontologies for the revision of beach nourishments. Int. J. Geogr. Inform. Sci., 19(10), 10571072.

Van der Wal, D. (2000). Modelling aeolian sand transport and morphological development in two beach nourishment areas. Earth Surface Processes and Landforms, 25, 77-92.

Van Noortwijk, J.M. and Peerbolte, E.B. (2000). Optimal sand nourishment decisions. J. Waterway, Port, Coast., Ocean Eng., 126(1), 30-38

Van Oort, P.A.J. (2006). Spatial Data Quality: From Description to Application, Ph.D. Dissertation, Wageningen University, The Netherlands, pp. 124.

Van Rijn, L.C. (1997). Sediment transport and budget of the central coastal zone of Holland. Coast. Eng., 32(1), 61-90.

Van Vuren, S., Kok, M. and Jorissen, R.E. (2004). Coastal defence and societal activities in the coastal zone: Compatible or conflicting interests?. J. Coast. Res., 20(2), 550-561.

Wilkie, D.S. and Finn, J.T. (1996). Remote Sensing Imagery for Natural Resources Monitoring, Colombia University Press, New York, pp. 295.

Yemefack, M. (2005). Modelling and Monitoring Soil and Land Use Dynamics within Shifting Agricultural Landscape Mosaic Systems, ITC Dissertation 121, Enschede, ITC, pp. 194. 